\title{
Atmospheric signatures by transit of HD209458 with VLT/UVES
}

\author{
Athena Coustenis ${ }^{1}$, N. Iro ${ }^{1,2}$, C. Moutou ${ }^{3}$, M. Mayor ${ }^{4}$ and D. Queloz ${ }^{4}$ \\ ${ }^{1}$ LESIA - Observatoire de Paris-Meudon, 5, place Jules Jannsen, FRANCE \\ email: athena.coustenis@obspm.fr \\ ${ }^{2}$ Department of Physics - University of Florida, USA \\ email: iro@phys.ufl.edu \\ ${ }^{3}$ LAM - Marseille, FRANCE \\ email: claire.moutou@oamp.fr \\ ${ }^{4}$ Observatoire de Genève, SWITZERLAND \\ email: Michel.Mayor@obs.unige.ch,Didier.Queloz@obs.unige.ch
}

\begin{abstract}
In our search for clues as to the nature of the exosphere of HD209458 (Moutou et al., 2001 ; Moutou et al., 2003, Iro et al., 2004), we have acquired VLT/UVES data during an ambitious observational campaign performed in June-September 2002 and covering 6 transits of the exoplanet. The resolving power was $\mathrm{R}=100000$ in the $0.475-0.68$ micron range. We search for ions and neutral molecules (such as $\mathrm{H} 2 \mathrm{O}+, \mathrm{CO}+, \mathrm{CH}+$, etc) originating in the planets exosphere and located in the evaporated material around the planet, occulting its primary star. We present in this paper a tentative search in the spectral regions where features of sodium or $_{2} \mathrm{H}^{+}$can be present.
\end{abstract}

Keywords. Exoplanets, transits, exosphere, atmosphere, techniques: spectroscopic, (stars:) planetary systems, line: identification.

\section{Introduction}

HD209458b, the first discovered extrasolar planet transiting its star, has been subject to several studies constraining the composition of its atmosphere (see Table 1), in particular Na (Charbonneau et al. 2002; Narita et al. 2005), HeI (Moutou et al. 2003), CO (Deming et al. 2005) , H (Bundy \& Marcy 2000; Vidal-Madjar et al. 2003; Vidal-Madjar et al. 2004; Winn et al. 2004; Narita et al. 2005), O, C and Si (Vidal-Madjar et al. 2004), Ca (Bundy \& Marcy 2000; Narita et al. 2005; Shkolnik et al. 2005) and Fe (Bundy \& Marcy 2000; Narita et al. 2005).

Our group has searched for such signatures since the first detection of 51 Pegasi from the ground and also from space with ISO (Coustenis et al. 1997, Rauer et al. 2000). We performed observations with UVES in the 0.33-0.67 $\mu \mathrm{m}$ region at high spectral resolution. We searched for ions and neutral molecules originating in the planet's exosphere and located in the evaporated material around the planet, occulting its primary star (Coustenis et al. 1997). Iro et al. (2004) presented the planet-induced chromospheric activity on HD209458 in these data as well as the He feature at $1.083 \mu \mathrm{m}$ with data from VLT/ISAAC (Moutou et al. 2003). In this paper, we focus on the search for features from $\mathrm{Na}$ and $\mathrm{H}_{2} \mathrm{O}^{+}$.

\section{The 2002 UVES campaign}

We used VLT/UVES to observe HD209458 in 2002 over 6 of its transits, thus accumulating the time required to obtain a $\mathrm{S} / \mathrm{B}$ of about 1000, required in order to detect 


\begin{tabular}{|c|c|c|c|c|}
\hline Constituent & & Position & Note & Reference \\
\hline \multirow[t]{2}{*}{$\mathrm{Na}$} & $\mathrm{D}$ & $5893 \AA$ & $<0.02 \%$ & Charbonneau et al. (2002) \\
\hline & $\begin{array}{l}\text { D1 } \\
\text { D2 }\end{array}$ & $5896.06 \AA$ & $<0.03 \%$ & Narita et al. (2005) \\
\hline $\mathrm{He}$ & $\mathrm{He} \mathrm{I}$ & $1083 \mu \mathrm{m}$ & $<05 \%$ & Moutoul et al (2001. 2003) \\
\hline \multirow[t]{8}{*}{$\mathrm{H}$} & $\operatorname{Ly} \alpha$ & $1215.67 \AA$ & $15 \%(1 \sigma)$ & Vidal-Madjar et al. (2003) \\
\hline & $\mathrm{H} \alpha$ & $6563 \AA$ & $<0.1 \%$ & Winn et al. (2003) \\
\hline & & & $<0.81 \%$ & Narita et al. (2005) \\
\hline & $\mathrm{H} \mathrm{I}$ & $1215 \AA$ & $5.3 \%(1 \sigma)$ & Vidal-Madjar et al. (2004) \\
\hline & $\mathrm{H} \beta$ & $4861.45 \AA$ & $<3.6 \%$ & Bundy \& Marcy (2000) \\
\hline & & & $<0.68 \%$ & Narita et al. $(2005)$ \\
\hline & $\mathrm{H} \gamma$ & $4340 \AA$ & $<2.7 \%$ & Bundy \& Marcy (2000) \\
\hline & & & $<1.57 \%$ & Narita et al. (2005) \\
\hline $\mathrm{O}$ & O I & $1305 \AA$ & $12.8 \%(1 \sigma)$ & Vidal-Madjar et al. (2004) \\
\hline $\mathrm{C}$ & C II & $1335 \AA$ & $7.5 \%(1 \sigma)$ & Vidal-Madjar et al. (2004) \\
\hline $\mathrm{Si}$ & Si IV & $1394 \AA$ & No detection & Vidal-Madjar et al. (2004) \\
\hline \multirow[t]{3}{*}{$\mathrm{Ca}$} & \multirow{3}{*}{$\begin{array}{l}\text { Ca II K } \\
\text { Ca II H } \\
\text { Ca II \& K } \\
\text { Ca I }\end{array}$} & \multirow{3}{*}{$\begin{array}{l}3943 \AA \\
3968 \AA\end{array}$} & Not quantified & Shkonlik et al. (2005) \\
\hline & & & $<14 \%$ & Bundy \& Marcy (2000) \\
\hline & & & $<2.14 \%$ & Narita et al. (2005) \\
\hline \multirow[t]{3}{*}{$\mathrm{Fe}$} & Fe I & $4384 \AA$ & $<3.3 \%$ & Bundy \& Marcy (2000) \\
\hline & & & $<1.08 \%$ & Narita et al. (2005) \\
\hline & Fe I & $6024.2 \AA$ & $<0.84 \%$ & Narita et al. (2005) \\
\hline $\mathrm{CO}$ & & $2 \mu \mathrm{m}$ & Very weak (no detection) & Deming et al. (2005) \\
\hline
\end{tabular}

Table 1. Detections of atmospheric constituents in HD209458b.

a possible evaporated exosphere. The observations were spread out between June and September 2002. An equivalent time of observation was dedicated to the observation of the system off-transit, to supply a spectrum of comparison of the primary star equivalent to the on-transit spectrum. Finally, a particular effort was devoted to the calibration of the event, at the same time by inserting frequent observations of a comparison star so as to insure the determination of the telluric contribution, and to provide a high-quality calibration of the instrument.

The data are in the course of analysis since the beginning of 2003. The production of a spectrum of transmission with no telluric or stellar contamination requires a meticulous reduction which we perform ourselves. We note the excellent quality of the majority of the spectra.

\section{Data analysis}

We averaged the spectra for all the transits in order to obtain ON-transit and OFFtransit spectra with a better $\mathrm{S} / \mathrm{N}$ ratio. We then calculate the difference between the ONand the OFF-spectra for the spectral ranges of interests. We note dissymmetric features in the region of $\mathrm{H}_{2} \mathrm{O}^{+}$lines at 6544 (see Fig. 1) and $6193 \AA$ (see Fig. 2) (the nominal position of the $\mathrm{H}_{2} \mathrm{O}^{+}$line is $6198 \AA$ ). Another region that we are investigating is the $\mathrm{Na}$ 


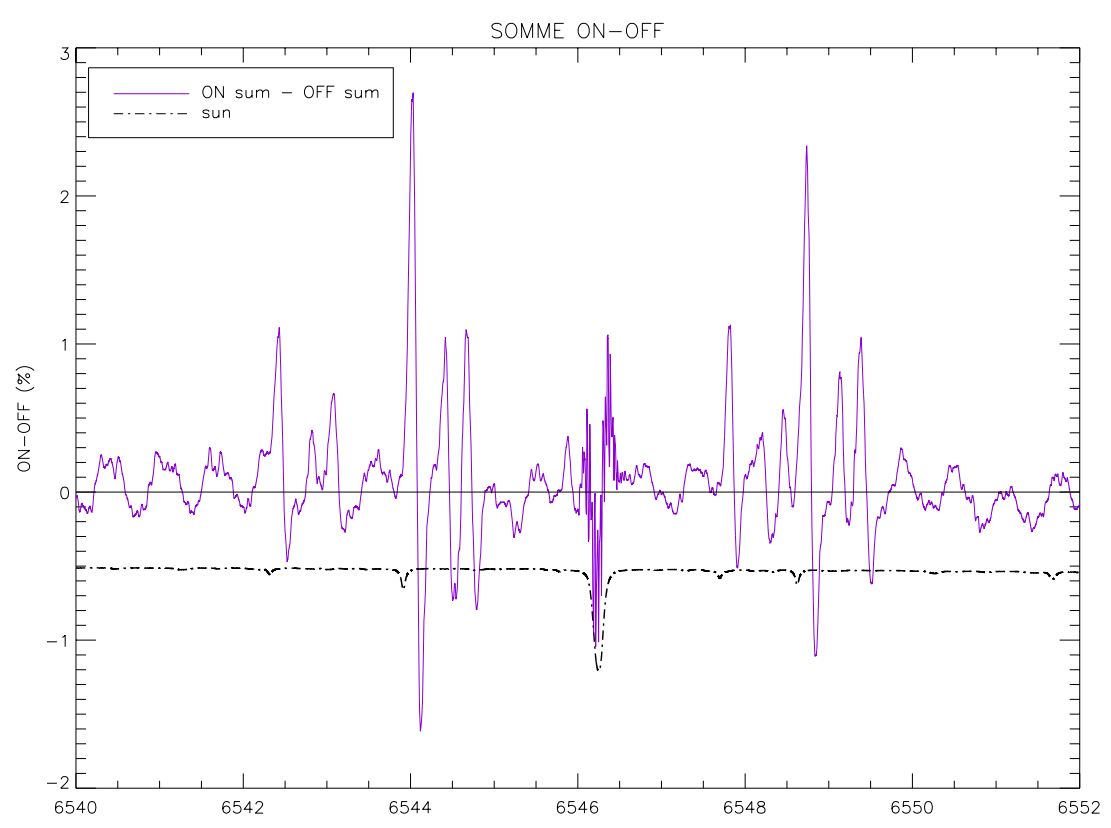

Figure 1. Difference between the sum of all the OFF-transit spectra and the sum of all the ON-transit spectra. The solar spectrum is shown in arbitrary units. We find a strong dissymmetry between $\mathrm{ON}$ and $\mathrm{OFF}$ at the $\mathrm{H}_{2} \mathrm{O}^{+}$line position at $6544 \AA$.

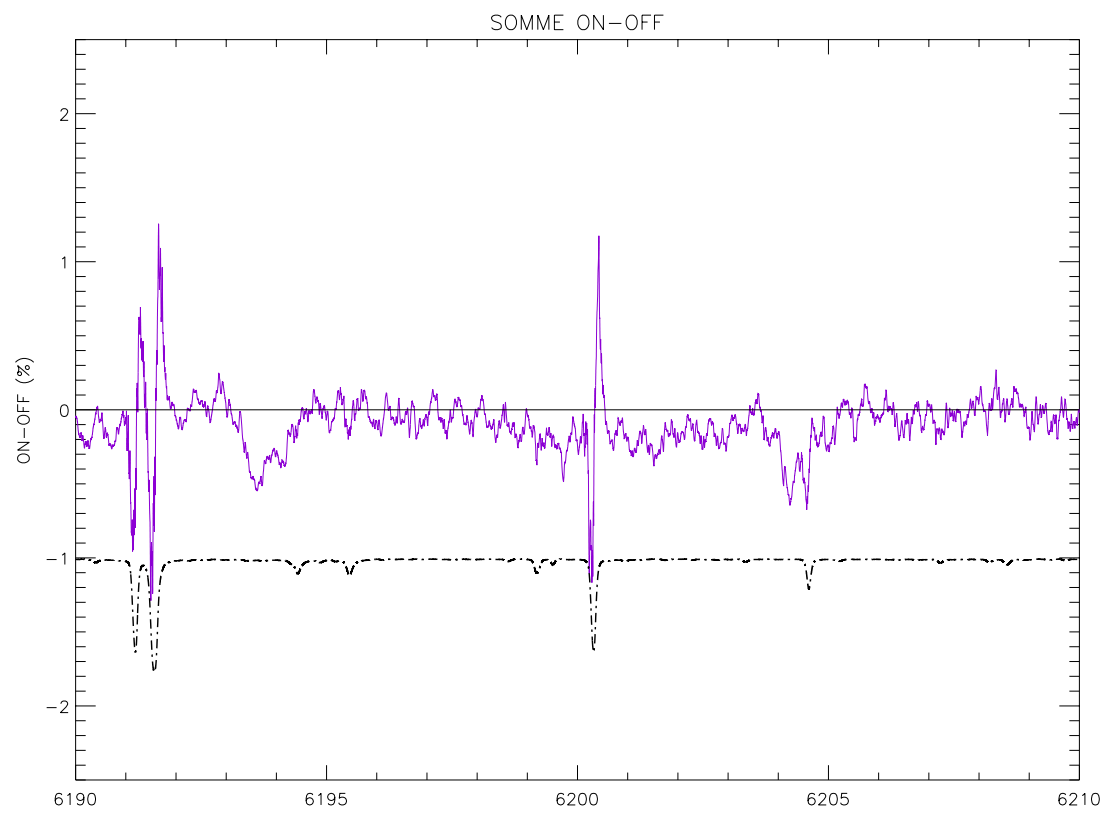

Figure 2. Search for $\mathrm{H}_{2} \mathrm{O}^{+}$(nominally at $6198 \AA$ ): zoom. The search in this region is complicated by the presence of a strong Fe line, but there seem to be unidentified features at 6193.5 $\AA$ and $6204.5 \AA$.

line doublet at 5891.9 and $5893.2 \AA$ where we note a dissymetry between the On- and the OFF- transit spectra. 


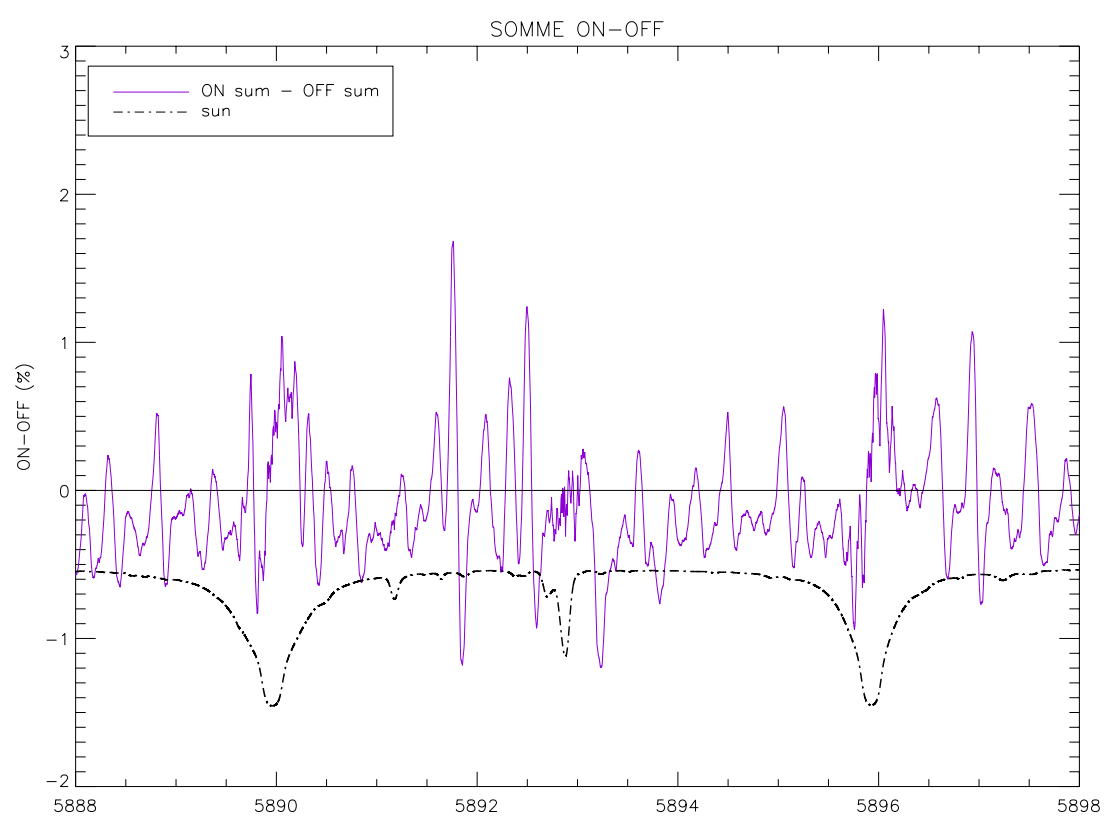

Figure 3. On- and off-transit spectra of HD209458 taken with UVES and the flux difference observed in two different orders within the $\mathrm{Na}$ band at $5893 \AA$ A. A zoom in the doublet region is shown, with a possible feature at $5893.2 \AA$.

\section{Perspectives}

The spectral ranges contained in the data presented in this paper include positions of possible features from $\mathrm{H} \alpha(6563 \AA)$ and $\mathrm{H} \beta(4861 \AA)$, Fe $(6024 \AA)$, O I $(5577$ and $6364 \AA), \mathrm{CO}^{+}(4911$ and $5490 \AA)$. The analysis of these spectral regions are in progress.

\section{References}

Bundy, K. A. \& Marcy, G. W. 2000, PASP 112, 1421

Charbonneau, D., Brown, T. M., Noyes, R. W., \& al. 2002, ApJ 568, 377

Coustenis, A., Scheider, J., Wittemberg, R., et al. 1997. ASP 134, 296-303

Deming, D., Brown, T. M., Charbonneau, D., et al. 2005, ApJ 622, 1149

Iro, N., Coustenis, A., Moutou, C., et al. 2004 in ASP Conf. Ser. Vol. 321, Extrasolar Planets: Today and Tomorrow, 209

Moutou, C., Coustenis, A., Schneider, J., et al. 2003. A \& A 405, 341.

Narita, N., Suto, Y., Winn, J. N., et al. 2005, PASJ, 57, 471

Rauer, H., Bockele-Morvan, D., Coustenis, A., et al. 2000, ApJ 622, 1075

Shkolnik, E., Walker, G. A. H., Bohlender, D. A., et al. 2005, A \& $A$ 355, 573

Vidal-Madjar, A., Lecavelier des Etangs, A., Désert, J.-M., et al. 2003, Nature 422, 143

Vidal-Madjar, A., Désert, J.-M., Lecavelier des Etangs, A., et al. 2004, ApJ Letters 604, L69

Winn, J. N., Suto, Y., Turner, E. L., et al. 2004, PASJ, 56, 655 